\title{
Museo y compromiso. Estudio de percepciones de estudiantes sobre una experiencia educativa
}

\section{Museum and engagement. Study of student perceptions of an educational experience}

DAIANA YAMILA Rigo*

FERNANDA MELGAR ${ }^{* *}$

Romina CECILIA ELISONDo***

El artículo presenta una experiencia educativa orientada a abrir espacios que sirvan de andamiaje al compromiso escolar. El estudio consistió en la planificación de una visita a un museo, acompañada de tareas previas y posteriores, pensadas para facilitar la participación y el interés del estudiantado en la propuesta y en los aprendizajes escolares que son parte de la experiencia. Participaron 53 estudiantes de tercer grado de una escuela primaria de Las Higueras (Córdoba, Argentina). Para la recolección de datos, se hicieron observaciones participantes y se administraron cuestionarios a los estudiantes. El análisis de datos arroja que estos se muestran interesados, con ganas de aprender, preguntar e interactuar con los objetos del museo, aspectos que potencian el compromiso. También se destaca el papel de los vínculos interpersonales y las emociones como aspectos positivos de la experiencia que incrementan los aprendizajes.

The paper presents a study of an educational experience aimed at generating spaces that support school engagement. Specifically, he planned a visit to a museum, accompanied by pre and post tasks, designed to facilitate the participation and interest of students regarding the proposal and the school learning that are part of the experience. The study was carried out in a primary school in Las Higueras (Córdoba, Argentina), involving 53 third grade students. For data collection, consult the participating studies and questionnaires were administered to the students. The data analysis shows that students consider themselves interested, eager to learn, ask questions and interact with the museum's objects, aspects that enhance engagement. The role of interpersonal ties and emotions as positive aspects of experience that enhance learning is also highlighted.

\author{
Palabras clave: \\ compromiso, \\ contextos no \\ formales, escuela, \\ experiencia \\ educativa
}

\section{Keywords:}

engagement, nonformal contexts, school, educational experience

Recibido: 13 de febrero de 2020 | Aceptado para su publicación: 6 de julio de 2020| Publicado: 16 de julio de 2020

Recuperado de: https://sinectica.iteso.mx/index.php/SINECTICA/article/view/1086 doi: 10.31391/S2007-7033(2020)0055-004

\footnotetext{
* Doctora en Psicología por la Universidad Nacional de San Luis. Investigadora asistente del Consejo Nacional de Investigaciones Científicas y Técnicas. Profesora del Departamento de Ciencias de la Educación en la Universidad Nacional de Río Cuarto. Línea de Investigación: compromiso y experiencias educativas. Correo electrónico: daianarigo@hotmail.com/ https://orcid.org/0000-0003-0312-6429

** Doctora en Psicología por la Universidad Nacional de San Luis. Ayudante de primera en el Departamento de Ciencias de la Educación en la Universidad Nacional de Río Cuarto. Docente de nivel superior en el Instituto Ramón Menéndez. Línea de investigación: museos y contextos no formales. Correo electrónico: fernandamelgar@gmail.com/https://orcid. org/0000-0003-4331-0319

*** Doctora en Psicología por la Universidad de Murcia y la Universidad Nacional de San Luis. Investigadora adjunta del Consejo Nacional de Investigaciones Científicas y Técnicas de Argentina. Profesora adjunta en el Departamento de Ciencias de la Educación de la Universidad Nacional de Río Cuarto. Línea de investigación: creatividad e innovación educativa. Correo electrónico: relisondo@gmail.com/https://orcid.org/0000-0002-7841-9878
} 


\section{INTRODUCCIÓN}

ensar en la fusión de contextos educativos, formales y no formales, es el desafío que enmarca los desarrollos teóricos de la psicología educacional, que postulan la importancia de complementar los entornos escolares con comunidades y programas educativos extraescolares (Corno \& Anderman, 2016). A este reto se suma el generar experiencias educativas que, al concretar esa unión, puedan ser pensadas como andamios para promover y apoyar el compromiso escolar de los estudiantes.

Bickmore, Pfeifer y Schulman (2011) plantean que los museos contemporáneos utilizan exhibiciones interactivas, multimedia, juegos, guías móviles automatizadas y otros mecanismos para entretener y comprometer a los visitantes a fin de que el aprendizaje tenga la oportunidad de darse, incluso mientras los estudiantes pasan de exhibición a exhibición. Hay autores que señalan cómo las visitas a museos contribuyen a ampliar los conocimientos de las personas, al incentivar la curiosidad y poder formular preguntas que les permiten aprender (Cabral y Maldonado, 2019). Los museos pueden ofrecer una variedad de posibilidades para aprender sobre disciplinas científicas y artísticas diversas.

Los museos se enfrentan a pruebas cada vez mayores en cuanto al diseño de nuevos espacios expositivos y su papel en la sociedad. En los últimos años se ha reconocido cada vez más que dichos lugares deben dar mayor prioridad a la experiencia del visitante y a las formas en que las áreas de exposición lo invitan a participar en la historia natural o cultural que desean contar (Dindler \& Iversen, 2009). Ese papel mayor en las interacciones con las exposiciones es remarcada por Bailey-Ross y colaboradores (2017) como un elemento central del compromiso público de los museos para incorporar actividades tendentes a promover el interés y la participación de las personas con los objetos y las propuestas de los museos.

El National Coordinating Centre for Public Engagement (NCCPE) señala que el compromiso público es un proceso bidireccional, que implica la interacción y la escucha con el propósito de generar aprendizajes a partir de una infinidad de formas en que las actividades y los objetos puedan ser compartidos y comunicados a las personas (NCCPE, 2015). Esa doble dimensión también puede pensarse para atender, de manera específica, el compromiso escolar, es decir, cómo el compromiso público de los museos puede, con base en sus propuestas y las experiencias que los estudiantes tienen, facilitar la implicación de ellos con lo ahí narrado.

En este artículo presentamos un estudio cuyo objetivo fue analizar las percepciones de niños y niñas sobre una experiencia educativa que tuvo lugar en un museo. Recuperamos las voces de los estudiantes participantes con el propósito de comprender las potencialidades de las visitas a museos como experiencias propicias para favorecer el compromiso escolar, y de crear conocimientos teóricos y prácticos que contribuyan a reconocer que esas visitas estimulan la enseñanza y el aprendizaje escolar. Nuestra intención es compartir conocimientos que aporten a futuras planificaciones y proyectos educativos más amplios en contextos educativos.

En la primera parte definimos posiciones teóricas que sustentan la planificación y el desarrollo de la experiencia educativa; articulamos museos y compromiso como 
ejes para pensar propuestas educativas innovadoras. La segunda parte contiene los aspectos metodológicos del estudio y la tercera, las consideraciones finales.

\section{CONSIDERACIONES INICIALES SOBRE COMPROMISO Y MUSEOS}

El compromiso es un metaconstructo potente, porque proporciona una imagen más rica de cómo los estudiantes piensan, actúan y sienten las experiencias educativas, y configura diversos patrones de compromiso como constructo maleable. La idea de experiencia educativa que aparece como dimensión contextual clave para promover la implicación de los estudiantes nos remite a la dimensión de las prácticas y las relaciones educativas que se tejen tanto dentro como fuera del aula, y lo que emerge de aquello que les pasa a los protagonistas de los aprendizajes frente a lo vivido. Estas vivencias modifican las manifestaciones afectivas, conductuales y cognitivas como dimensiones que median el quehacer pedagógico y los significados construidos (Fredricks, Reschly \& Christenson, 2019; Rigo y Donolo, 2019; Contreras y Pérez, 2010).

El compromiso es un proceso que evoluciona en el tiempo como respuesta a las interacciones de los sujetos y las características de los entornos educativos donde ocurren sus aprendizajes. Archambault, Janosz, Goulet, Dupe're' y Gilbert-Blanchard (2019) sostienen que es la manera en que se va conjugando la educación que reciben los niños como motor para pensar el compromiso a lo largo de la escolarización, la clave para comprender por qué algunos permanecen implicados y una alta proporción se va desvinculando desde la entrada a la escuela en adelante.

Esta preocupación inquieta a los educadores e investigadores, los incita a pensar no solo en maneras renovadas de diseñar el aula, sino también de ampliar los contextos educativos. Podemos identificar una gran variedad de intervenciones que tienen como foco incrementar el compromiso de los estudiantes. Estas intervenciones difieren en términos de metas, objetivos y población atendida. Inicialmente, fueron diseñadas con el objetivo de reducir las tasas de deserción escolar, centradas en los factores individuales que ponen a los estudiantes en riesgo de abandonar la escuela. Con el tiempo, se advierte un cambio hacia una mayor atención en los factores contextuales que pueden ayudar a promover la implicación escolar y su finalización. El objetivo de estos programas es aumentar el compromiso de los estudiantes al abordar los factores contextuales que pueden ayudar a los estudiantes a adquirir los aprendizajes escolares y crear las relaciones, los contextos y los recursos necesarios para convertirse en participantes activos en la escuela (Fredricks, Reschly \& Christenson, 2019).

La perspectiva del medio ambiente más la persona, de Eccles y Midgley (1989), es promisoria para estudiar el compromiso y configurar experiencias para facilitarlo. Esta perspectiva señala que el compromiso se verá influenciado por el ajuste entre las necesidades y los objetivos de una persona y las oportunidades disponibles para satisfacerlas y las metas en su entorno. Con base en este modelo, las investigaciones se han centrado cada vez más en las características de las actividades específicas y en los entornos en los cuales ocurren, y cómo el compromiso con esas tareas y contextos se asocia a desarrollos positivos (Bayat, 2015; Fletcher, Nickerson \& Wright, 2003; Fredricks \& Eccles, 2008; O’Brien \& Rollefson, 1995).

Respecto a las características de las actividades que podrían ayudar a promover el compromiso de los estudiantes, Blumenfeld, Kempler y Krajcik (2006) entienden

Rigo / Melgar / Elisondo. Museo y compromiso. Estudio de percepciones de estudiantes sobre una experiencia educativa Sinéctica 55 www.sinectica.iteso.mx 
que tanto el valor intrínseco como instrumental deben estar presentes. También, reconocen como necesarias las posibilidades de colaborar y poder tomar decisiones referidas, por ejemplo, al nivel de dificultad. Las posibilidades de colaborar pueden materializarse al ofrecer ocasiones para preguntar, compartir y discutir ideas, así como usar tecnologías que permitan mejorar y ampliar los aprendizajes.

Los estudios sobre contextos extraescolares o no formales, como los denomina Eastwell y Rennie (2002), se utilizan para describir el proceso educativo fuera de la escuela. Morentin y Guisasola (2014) mencionan que las salidas escolares son experiencias que pueden mejorar el aprendizaje, promover el interés y complementar la tarea escolar.

Templeton y Baskinger (2011) afirman que las visitas a los museos para promover el compromiso de los estudiantes tienen que estar ligadas a experiencias resonantes, ricas e interactivas. La resonancia ocurre cuando un objeto o evento trae recuerdos de vivencias, pensamientos o sentimientos personales. La riqueza se logra cuando la información que se brinda va más allá de una lista de hechos y se habilitan formas variadas de presentar la información disponible para despertar la curiosidad de los visitantes. La interactividad se alcanza cuando el visitante explora por su cuenta las exposiciones y gana mayor autonomía y flexibilidad en el recorrido vinculado a preferencias o intereses personales. Alderoqui y Pedersoli (2011) las llaman experiencias memorables. Asimismo, el museo ofrece una variedad de ayudas interpretativas para involucrar a los sujetos, por ejemplo, folletos, audioguías, guías docentes y etiquetas de pared. Estas ayudas resultan limitadas y pueden ampliarse si ofrecen a los visitantes posibilidades de experimentar.

Desde el punto de vista del entorno, los museos pueden considerar diferentes aspectos para promover experiencias memorables en los niños. Siguiendo el modelo contextual del aprendizaje (Falk \& Dierking, 2000), son esenciales los aspectos del contexto físico, social y personal. En general, un elemento que colabora en la construcción de conocimientos es la experiencia directa y la manipulación de objetos según los intereses y las necesidades de los niños; también la exploración del ambiente por medio de los sentidos. Desde el punto de vista social, es necesario asegurar ocasiones en las que se puedan emplear los recursos individuales, sociales y culturales para lograr metas, y reconocer el papel significativo de los padres y maestros. Desde el punto de vista personal, resulta conveniente trabajar de manera explícita en el desarrollo de respuestas afectivas que incluyan intereses, actitudes y emociones de los niños (Melgar, 2019).

Lo cierto es que más allá del entorno y sus rasgos, las personas también buscan motivos para comprometerse con objetos y contextos diversos. Reeve, Cheon y Jang (2019), en una contribución reciente, señalan que el compromiso refiere a la participación activa de un estudiante en una actividad de aprendizaje, dirigida por objetivos y propósitos específicos, entre los cuales mencionan el conseguir mejores logros educativos, satisfacer sus motivaciones y crear entornos de aprendizaje favorables y afines a sus intereses.

La relación museos y compromiso puede ser analizada a la luz de diferentes estudios. Unal (2012) ha observado que los niños permanecen más tiempo y formulan más preguntas en áreas de los museos que son de su interés. Anderson, Piscitelli, 
Weier, Everett y Tayler (2012) han destacado la diversidad de intereses y recuerdos que dan cuenta del carácter único de la experiencia en museos para cada sujeto. Las investigaciones subrayan la relevancia de las experiencias previas (Castro, 2003) y de los vínculos afectivos que surgen con otros niños y con los educadores (Chávez, 2015).

En general, los estudios indican que los niños muestran interés por las propuestas de los museos, en especial por aquellas que suponen interacciones con los objetos (crear, tocar, jugar y experimentar), y el deseo de los participantes por realizar nuevas visitas. Los niños también sugieren actividades que pueden ser atractivas en las visitas a los museos, por ejemplo, la danza, las obras de teatro y la elaboración de artesanías (Chávez, 2015).

En el estudio de Melgar (2019), cuyo objetivo fue conocer y analizar las percepciones y experiencias de niños en museos, participaron 133 niños y niñas ubicados en la franja etaria de los ocho a los trece años de escuelas de gestión pública y privada, rurales y urbanas, de la ciudad de Río Cuarto, Argentina. Los resultados se presentan en tres categorías: percepciones sobre los museos, experiencias previas y valoraciones de una actividad particular. En general, los museos fueron percibidos de manera positiva por los niños como lugares para aprender, conocer, entretenerse, divertirse y salir del aula. De igual modo, valoraron como favorables sus experiencias en el museo y reconocieron diversos aprendizajes construidos a partir de la visita.

En suma, los museos se constituyen en espacios propicios para vivenciar experiencias significativas de enseñanza y aprendizaje. Asimismo, pueden promover el compromiso al ser contextos que facilitan la interacción, los vínculos entre sujetos y la construcción de conocimientos sobre diferentes objetos e historias. En nuestro estudio nos interesa analizar, desde las voces de algunos niños y niñas, las potencialidades educativas de una visita escolar a un museo.

\section{Metodología}

Nuestra investigación tiene un diseño descriptivo que combina análisis cuantitativos y cualitativos con el objetivo de comprender las perspectivas de los participantes. Nos centramos en una experiencia particular; no buscamos la generalización de los resultados, sino el estudio preliminar de algunas percepciones de los niños y las niñas sobre la visita y el compromiso.

\section{Participantes y contextos}

Nuestra investigación se llevó a cabo en el contexto de una actividad diseñada por educadoras e investigadoras de la Universidad Nacional de Río Cuarto. En la propuesta participaron docentes del espacio curricular obligatorio denominado ciencias sociales de una escuela pública y de nivel primario de la provincia de Córdoba, Argentina. La tarea tuvo la intención de vincular los contenidos de las instituciones con una visita al Museo Tecnológico Aeroespacial. En el estudio participaron 53 estudiantes que, al momento de la visita, cursaban el tercer grado de la escuela primaria, turno matutino, de dos divisiones; sus edades iban de los siete a los ocho años de edad; los mayores constituían casi la totalidad. 
Atendimos los aspectos éticos y tomamos las precauciones necesarias para resguardar el anonimato y la confidencialidad de los datos, a partir de la solicitud del asentimiento informado a los padres o tutores de los estudiantes.

\section{Descripción de la experiencia}

La experiencia educativa quedó estructurada en tres momentos. El primero consistió en una actividad en el aula antes de la visita al museo. Solicitamos a los estudiantes que pensaran y dibujaran su objeto preferido y mencionaran el significado que tiene para ellos. La actividad se acompañó de una lista de preguntas orientadas a conocer las percepciones sobre qué es un museo, cuáles son las actividades que se pueden realizar en esas instituciones, para qué se le visita, cuándo y sus experiencias previas en torno a esta institución. Todas las preguntas ofrecían alternativas cerradas como modalidad de respuesta, excepto la primera, la que demandaba una respuesta abierta. Las respuestas de los estudiantes fueron parte de una puesta en común antes de visitar el museo.

En un segundo momento, los estudiantes, junto con las maestras y los especialistas, visitaron el museo, ubicado en un predio que cuenta con amplios espacios verdes, que se conjugan con los galpones que forman parte del Área de Material Río Cuarto (Unidad de la Fuerza Aérea Argentina). Funciona con tres muestras permanentes que cuentan la historia del área y de la aviación en el mundo, así como las características físicas del aire. Durante el recorrido guiado, los niños registraron los lugares por los que iban pasando y aquello que veían.

En un tercer momento, en el ámbito del aula y con base en sus registros, los estudiantes relataron sus experiencias, vivencias y aprendizajes. Se configuró, así, un contexto para compartir las diferentes narrativas.

\section{Instrumentos de recolección de datos}

Para la recolección de datos, empleamos un cuestionario y las observaciones participantes. El cuestionario estuvo organizado en torno a siete preguntas que requerían respuestas abiertas, además de la edad, el grado y la división escolar. Las preguntas se orientaron a saber cómo percibían los niños las actividades de aprendizaje y sus momentos. También, consideramos la vinculación con el compromiso, en términos de la participación y el interés por la propuesta y los aprendizajes logrados. Las preguntas indagaron lo que más y menos les gustó de la visita al museo, los espacios y momentos para formular preguntas, las consultas realizadas, lo que modificarían de sus experiencias y si regresarían al museo en una nueva oportunidad. Finalmente, les pedimos una frase que resumiera el significado de la experiencia en el Museo Tecnológico Aeroespacial.

Mediante la observación participante, obtuvimos datos sobre los diferentes momentos de la visita escolar, los intercambios producidos entre los estudiantes, las docentes y los educadores del museo. Los diálogos fueron registrados con base en las grabaciones. 


\section{Análisis de datos}

Recurrimos a un programa estadístico de libre acceso para el análisis cuantitativo de datos. Los análisis cualitativos para la construcción de categorías se realizaron con procesos de codificación y comparaciones permanentes. Triangulamos análisis cuantitativos y cualitativos para la comprensión de las percepciones de los estudiantes antes, durante y después de la experiencia.

\section{RESULTADOS}

\section{Antes de la visita}

En momentos previos a la visita, encontramos que los estudiantes mostraron diversas percepciones acerca de los museos: desde espacios para conservar una variedad de objetos hasta lugares donde se guardan piezas antiguas. La mayoría los señaló como entornos de aprendizaje: "enseñan con cosas antiguas", "ver cosas conocidas o desconocidas para aprender", "un lugar donde hay cosas muy pero muy antiguas y para aprender", "aprender mucho sobre muchas cosas interesantes", entre otras expresiones.

Gran parte de los estudiantes coincidieron en que el museo es un lugar donde se aprende, se disfruta, se cuentan historias y se sienten emociones diversas (45 sujetos, 85\%); asimismo, lo perciben como un espacio donde se debe guardar silencio (40 sujetos, 75\%). Otro grupo lo consideró como un área para entretenerse y ver objetos relacionados con el arte, como pinturas, cuadros o esculturas (20 sujetos, $38 \%$ ). En menor medida, lo concibieron como espacio de entretenimiento (19 sujetos, $36 \%$ ), para partir pronto (11sujetos, $21 \%$ ), aburrirse (8 sujetos, $15 \%$ ) o cansarse ( 5 sujetos, 9\%). Cabe destacar que ningún alumno o alumna optó por la opción del museo como un lugar para no ir.

En otros estudios, Melgar (2019), Castro (2003) y Chávez (2015) han observado que, en general, los niños perciben los museos como lugares vinculados a objetos y cosas relacionados con la historia y el pasado. También los consideran "importantes y valiosos para nosotros" y donde es posible conocer, aprender, observar y disfrutar.

Un aspecto que llama la atención es que este grupo (de tercer grado), a diferencia de otro (de sexto grado) que participó en un estudio anterior (Melgar, 2019), señaló con mayor frecuencia que el museo es un lugar donde hay que guardar silencio. Tal vez este señalamiento tenga que ver con las interacciones promovidas con docentes y educadores de los museos. A veces, cuando los grupos son grandes, pero de edades pequeñas, suelen destacarse las reglas y normas, más propias del espacio escolar.

Respecto a la utilidad del museo, la relacionan con el aprendizaje y el conocimiento (45 sujetos, $85 \%$ ); en seguida con la diversión o la ocasión de salir de la escuela (28 sujetos, 53\%); y con el aburrimiento o la distracción (8 sujetos, $15 \%)$. También indican que visitan los museos como parte del plan de vacaciones (25 sujetos, 47\%), por la escuela (17 sujetos, $32 \%$ ), por algún viaje y en compañía de un adulto ( 5 sujetos, $9 \%$ ). Finalmente, solo una minoría afirmó que conoce otros museos (11 sujetos, $21 \%$ ). 
En cuanto al objeto preciado dibujado, los estudiantes destacaron regalos significativos de un ser querido en la primera infancia que asocian con la emoción, alegría y recuerdos positivos; o bien, obsequios más cercanos en el tiempo, como los referidos al día de niños, realizados por sus padres, en su mayoría juguetes, que vinculan a la diversión.

Los objetos se constituyen en hitos en torno a los cuales vamos configurando nuestra subjetividad. Reservorios y refugios emocionales sobre los que generamos señas de identidad. Necesitamos verlos de vez en cuando -los guardamos en sitios especiales con mimo- o permanentemente -los exhibimos en la "colección particular" de nuestros museos familiares: dormitorio, salón, etcétera- (Melgar y De los Reyes, 2018).

La relación entre los objetos personales significativos y los museos remite a la idea de ofrecer soportes para pensar por qué estas instituciones se encargan de conservar, investigar y comunicar aspectos referidos a objetos patrimoniales. De ahí la insistencia en hacer explícitas las vinculaciones entre objetos personales y objetos simbólicos en los museos. En ambos casos, sirven para relatar historias que forman parte de nuestra subjetividad.

\section{Después de la visita}

De la experiencia educativa en el museo, concretamente, los estudiantes resaltan varios motivos para comprometerse. Con base en el análisis de los datos, podemos identificar tres categorías que muestran cómo los estudiantes que asistieron y participaron en la experiencia educativa se comprometieron con las exposiciones del museo, expresadas en diversión y aprendizaje, formular más preguntas y experimentar.

\section{Diversión y aprendizaje}

Los estudiantes rescataron de la experiencia los aspectos que pudieron identificar con una doble finalidad: no solo les causaban interés, sino que, además, los estimulaban a aprender más. A modo de ejemplo, compartimos algunas respuestas:

La experiencia fue divertida y única.

Me gustó todo lo que vi y aprendí.

Es divertido y se aprende mucho.

El museo fue lindo para aprender.

El museo fue divertido.

Me parecía divertido, fantástico y genial.

Nos explicaron muchas cosas que no sabíamos. 
En el museo aprendí todo.

En el museo aprendí más.

Fue una experiencia educativa e histórica.

Las percepciones de los estudiantes revelan que al museo no solo lo vivencian como fuente de información y un lugar para aprender y pasarla bien; también estos fueron los principales motivos que los estudiantes indican como factores que promovieron su implicación con la visita y el recorrido realizado. Sobre estos dos componentes, aprendizaje y disfrute, Shernoff (2013) describe que, para comprometerse, no es suficiente solo disfrutar (play-like engagement), sino, ante todo, percibir desafíos (work-like engagement); la combinación de ambos factores define, además, los contextos propicios para introducir a los estudiantes en nuevos escenarios de aprendizaje (Rigo y Donolo, 2018).

En cuanto al aprendizaje que ocurre en el museo, Alderoqui y Linares (2005) exponen que un buen indicador de aprendizaje en la exposición son los comentarios relativos a los contenidos y los vínculos que se tejen. Al respecto, identificamos que los estudiantes fueron enlazando el contenido con experiencias personales ("mi papá hizo el servicio militar acá", "me hizo acordar mucho a mi tío"), con expresiones de satisfacción ante un desafío propuesto ("qué difícil es hacer, reparar y manejar un avión"), con comentarios referidos a conexiones con objetos y aprendizajes previos o relacionados (“¿ese avión se usó en la Guerra de Malvinas?" y con preguntas como indicador de atención sostenida para solicitar mayor información ("cuántos soldados vivían acá?, ¿de dónde venían?, ¿todos esos aviones se repararon acá?"), entre otras.

Las voces de los estudiantes ponen en evidencia que el recorrido despierta emociones, recuerdos y aprendizajes previos que vuelven a significarse a la luz de nuevos contenidos y redes conceptuales que se tejen en el entramado de las visitas, entre estudiantes, educadores de museos y docentes.

De acuerdo con Anderson, Piscitelli, Weier, Everett y Tayler (2002), las experiencias de los niños en los museos se caracterizan por la diversidad de intereses y la naturaleza individualista e idiosincrásica de los recuerdos y los aprendizajes; es decir, cada niño o niña se vincula de manera particular con los objetos y las historias de los museos a partir de sus experiencias personales y familiares. Según estos autores, con base en los vínculos entre lo que propone el museo y las experiencias de los niños, se construyen y reconstruyen aprendizajes, emociones e intereses, de ahí que la experiencia de aprendizaje en los museos se conciba como situada y contextualizada.

De manera similar, en un estudio anterior, los niños percibieron los museos, en su mayoría, como contextos para aprender y conocer. Lejos de considerarlos como aburridos, señalaron que en los museos pueden divertirse y distraerse (Melgar, 2019). Unal (2012) también destaca emociones positivas de los niños durante las visitas a museos. En específico, observó que la mayoría de estos reaccionaban con risas ante algunos de los objetos del museo. Los participantes demostraron un marcado interés por ciertos objetos, deseos de conocer y búsqueda de articulaciones con experiencias personales y familiares. 
Hacer preguntas y más preguntas

Lo cierto es que los estudiantes, en su mayoría, reconocen que durante el recorrido en el museo se habilitaron numerosos espacios para hacer preguntas y consultas, como posibilidades de intercambiar con la educadora del museo, con las docentes o sus compañeros. Mucho más interesante es que gran parte de ellos volverían al museo para seguir indagando sobre aspectos que quedaron en el tintero por falta de tiempo. A modo de ejemplo, compartimos algunas respuestas:

Para aprender mucho más.

Cuando no entendía algo.

Para aprender más.

Volvería para aprender más.

Sobre los carteles, porque no entendía (línea del tiempo).

Me animé a algo que nunca hice.

Volver para hacer preguntas que no pude hacer..., no me dio el tiempo.

Sí, volvería para preguntar más.

Del mismo modo, Unal (2012) observó que los niños sentían curiosidad acerca de muchos objetos en el museo. Esa curiosidad se materializó en múltiples y variadas preguntas acerca de las funciones y el contexto de uso de los objetos. Según la autora, los niños demandan información para comprender y vincular lo percibido con sus experiencias previas; además, subraya el valor de las respuestas y los apoyos que puedan brindar los adultos para potenciar aprendizajes a partir de las preguntas de los niños.

El preguntar y las preguntas que se formularon son considerados por Alderoqui y Linares (2005) como un componente central del aprendizaje en museos y del compromiso o la participación explicativa de los visitantes. Estos autores señalan que las conversaciones durante las visitas son una medida del grado en el cual los visitantes se involucran en oportunidades explicativas particulares. De hecho, Rigo y Donolo (2019) puntualizan que el hacer preguntas es un indicador del compromiso conductual de los sujetos hacia aquello que es objetivo de conocimiento, aprendizaje y disfrute.

Leinhardt y Crowley (1998) distinguen cuatro niveles de compromiso explicativo: de lista o catalogación, de análisis, de síntesis y de explicación. De acuerdo con estas ideas, podríamos afirmar que los estudiantes, en su mayoría, formularon preguntas simples, de lista o catalogación, que intentaban conocer sobre las características de los objetos que se exponen en el museo; por ejemplo, ¿por qué hay un soldado con traje azul?, ¿de qué está hecha la ropa?, ¿cómo se hizo la comida?, ¿por qué ese espacio está libre, se fue el soldado?, entre otras. Los estudiantes también se plantearon preguntas sobre el nivel de análisis, aquellas que indagaban acerca de las características ocultas de los objetos; de síntesis, para integrar conocimientos, contenidos o ideas; y de explicación, orientadas a ampliar la comprensión de cómo funciona algo o cómo sucede tal cosa. 
Cabe destacar que las preguntas de explicación aparecieron en menor medida. A modo de ejemplo, compartimos algunas respuestas:

¿Por qué hay números arriba de la sala? ¿Cuántos aviones arreglaron? ¿Por qué los muñecos tienen rasgos distintos? ¿Cómo se llama este espacio? Cuando se dice 1901, ¿no era este espacio? ¿Acá en 1944? ¿Acá vivían los 400 soldados? ¿Por qué acá en Las Higueras, la base? ¿Cómo se maneja el simulador?

Estas preguntas dan indicio de que los estudiantes están ocupados, entretenidos, atentos e involucrados con la visita al museo, un compromiso que se extiende a futuras visitas. Asimismo, muestran que el aprendizaje es resultado de la interacción social que se da en el museo producto de las interpretaciones, las consultas y las explicaciones entre los alumnos, los docentes y la guía. Las preguntas son señales de la actividad mental de los estudiantes; los sujetos son activos en la construcción de conocimientos.

Las preguntas que quedan en el tintero y animan una nueva visita revelan que las exposiciones e interacciones que ocurren durante la visita no responden siempre a la totalidad de las dudas o consultas, lo que provoca nuevas búsquedas. Sería interesante que los museos, principalmente aquellos que realicen actividades con niños, abran espacios para preguntar, trabajar sobre las preguntas, relacionarlas con acciones que fueron realizadas o que podrían llevarse a cabo.

Las preguntas deberían propiciar comprensiones que permitan ir descubriendo las relaciones dinámicas fuertes entre palabras y acción; palabras, acción y reflexión (Freire y Faundez, 2013). Los museos constituyen espacios para la curiosidad y la interrogación. La diversidad de objetos culturales y de relatos habilita la potencialidad de la pregunta (Melgar, Elisondo y Donolo, 2018). La curiosidad suele estar ligada a la capacidad de asombro y a la novedad. Desde la perspectiva de la neurociencia, Ballarini (2015) asegura que la novedad tiene un papel clave en el proceso de aprendizaje, a tal punto que, sin el efecto de la novedad, muchas memorias jamás se consolidarían y se perderían por siempre en el olvido.

\section{Experimentar a través de la interacción}

Los estudiantes señalaron como sus "zonas favoritas" aquellas donde fue posible interactuar, tocar y experimentar. Se resalta la importancia de los espacios interactivos para aprender junto con las explicaciones ofrecidas por docentes o los educadores del museo: "subir al avión y visitar la cocina", "cuando subimos al avión", "lo que más me gustó fue subir al avión y simular un vuelo", "subirme al simulador de avión” y "aprendí interaccionando con los aviones y la guía”.

Cabral y Maldonado (2019) mencionan que los museos cada vez más interactivos facilitan y complementan el aprendizaje de los contenidos comentados en las aulas, e incentivan la curiosidad y el deseo de preguntar. Domínguez (2003) agrega que la interactividad fomenta la comunicación y la capacidad participativa, lo que permite al estudiante no solo recibir mensajes expositivos, sino pasar de sujeto pasivo a sujeto activo y ser artífice de su propio aprendizaje.

La experiencia analizada es congruente con la definición de museo como un lugar de encuentro, relaciones sociales, diálogo, descubrimientos y aprendizajes. Estas 
concepciones van permitiendo una reestructuración de las ideas preliminares de los estudiantes sobre qué es un museo; así se destacan expresiones acerca de la visita como algo "hermoso", "divertido", donde "aprendí mucho" y "volvería".

La satisfacción con la experiencia también puede verse reflejada en la idea de que los estudiantes no identificaron momentos no placenteros o no gratos. En este sentido, algunas frases fueron "no cambiaría nada, todo me gustó", "nada, estuvo muy bueno", "no cambiaría nada".

\section{CONSIDERACIONES FINALES}

Los análisis indican que los museos son contextos promisorios para ampliar las posibilidades que tienen los estudiantes de comprometerse con la enseñanza y el aprendizaje escolar. Las expresiones y percepciones de los niños muestran que es posible construir muchos y variados conocimientos a partir de las interacciones con los objetos y los sujetos que habitan los museos. También resulta interesante destacar el papel de los vínculos y las emociones como aspectos positivos de las visitas que potencian los aprendizajes. Ver, tocar, sentir, estar con otros, "subirse al avión", son acciones que se generaron gracias a la visita y que fueron valoradas positivamente por los niños.

Asimismo, los museos despiertan preguntas, proceso cognitivo básico para el aprendizaje, la creatividad (Corbalán, 2008) y el compromiso (Rigo y Donolo, 2018). Las preguntas emergentes de las visitas pueden ser retomadas y reconstruidas en las aulas a fin de crear oportunidades de articulación transversal entre diversos contenidos curriculares. Entonces, los museos son contextos promisorios de enseñanza y aprendizaje en tanto permiten ver, escuchar, estar con otros, vivenciar experiencias significativas y hacer preguntas. Contribuyen a pensar la educación como variada e ilimitada en formas, espacios, recursos y motivaciones, en pos de promover el compromiso de los estudiantes (Rigo y Donolo, 2018).

Coincidimos con Unal (2012) en que los museos ofrecen oportunidades para que los niños desarrollen aprendizajes a través de la investigación, la exploración, el cuestionamiento, la curiosidad, la creatividad, el uso de conocimientos previos y comparaciones, y el desarrollo de pensamientos críticos. Castro (2003) también destaca la importancia de la creatividad y el pensamiento divergente en los procesos educativos en museos. El autor considera indispensable que

cada visitante pueda estar claramente involucrado en un proceso genuinamente pedagógico y participativo que lo haga realmente un activo y dinámico protagonista del museo, por fuera del esquema del antiguo visitante que se limitaba a aceptar el discurso museal sin cuestionarlo siquiera y a actuar de manera pasiva y desinteresada frente a "verdades absolutas", producto del saber de unos pocos (p. 9).

En este sentido, vale la pena resaltar las preguntas de los niños y sus interpretaciones novedosas sobre los objetos y las historias que se cuentan en los museos.

Los resultados del estudio son interesantes a la luz del estudio anterior de Melgar (2019); si bien las planificaciones de las experiencias fueron diferentes (una de ellas fue diseñada en especial para los niños desde el museo y la otra fue una visita educativa propuesta desde la escuela), las percepciones de los niños son muy similares. En 
ambas experiencias reconocen valiosas potencialidades educativas de los museos, identifican acciones y aprendizajes construidos, y subrayan el papel de las emociones. En nuestro estudio y en el de Melgar (2019) se hace hincapié en esta poderosa afirmación: “¡Todo me gustó!”, expresión que sintetiza la valoración de la mayoría de los participantes; a la vez, son palabras que interpelan e invitan a pensar en la necesidad de más experiencias más allá de las aulas para potenciar el compromiso de los estudiantes.

En suma, las percepciones de los estudiantes sobre el museo como experiencia educativa que compromete se resumen en expresiones relacionadas con el interés, el deseo de aprender, preguntar e interaccionar con los objetos del museo, aspectos que fomentan el compromiso de los estudiantes, como lo puntualiza el estudio de Rigo (2017) y los planteamientos de Rigo, De la Barrera y Elisondo (2018a).

Cuatro palabras: interesante, desafiante, interrogantes e interacción, marcan la experiencia educativa de recorrer el Museo Tecnológico Aeroespacial, en la cual el compromiso de los estudiantes hacia la visita queda ligada a una experiencia de aprendizaje resonante, en los términos de Templeton y Baskinger (2011), evocada en recuerdos y emociones personales; rica a nivel cognitivo, al aprender nuevas ideas y generar preguntas novedosas en los visitantes; e interactiva en la posibilidad de no solo ver y escuchar, sino de aprender de acuerdo con los objetivos que invitan a su exploración activa.

En futuras investigaciones sería interesante incluir entrevistas y grupos focales para recuperar las voces de los niños. Asimismo, incorporar las expresiones de los docentes y articularlas con las valoraciones de los niños. En próximos estudios será importante también comparar resultados de diversas experiencias en museos con niños (visitas escolares, visitas con familiares, actividades planificadas por los museos, etcétera).

Los museos permiten aumentar la educación (Elisondo, 2015), es decir, generar mayores ocasiones para construir conocimientos e historias con otros y con objetos de la cultura. De igual modo, posibilitan ampliar miradas y horizontes; aprovechar estas ocasiones de potenciar los aprendizajes es un importante desafío para las escuelas. Construir muchos y variados conocimientos, en contextos heterogéneos dentro y fuera de las aulas, es indispensable para ampliar las oportunidades educativas de todos los sectores y fortalecer el compromiso (Rigo, De la Barrera y Elisondo, 2018b). Muchos conocimientos y muchas experiencias para todos los sujetos y a lo largo de la vida son imprescindibles para "garantizar una educación inclusiva, equitativa y de calidad y promover oportunidades de aprendizaje durante toda la vida para todos". Este es el cuarto objetivo de la Agenda 2030 de la Organización de las Naciones Unidas para la Educación, la Ciencia y la Cultura (Unesco, 2016).

\section{REFERENCIAS BIBLIOGRÁFICAS}

Alderoqui, S. y Linares, M. C. (2005). El libro de visitantes del museo de las escuelas: un diálogo entre narrativas. Enseñanza de las Ciencias Sociales, vol. 4, pp. 117-128. Recuperado de https://www.raco.cat/index.php/EnsenanzaCS/article/view/126256

Alderoqui, S. y Pedersoli, C. (2011). La educación en los museos. De los objetos a los visitantes. Buenos Aires: Paidós. 
Anderson, D., Piscitelli, B., Weier, K., Everett, M. \& Tayler, C. (2002). Children's museum experiences: Identifying powerful mediators of Learning. Curator the Museum Journal, vol. 45, núm. 3, pp. 213-231. Recuperado de https:// eprints.qut.edu.au/19073/.

Archambault, I., Janosz, M., Goulet, M., Dupe're', V. \& Gilbert-Blanchard, 0. (2019). Promoting student engagement from childhood to adolescence as a way to improve positive youth development and school completion. En J. Fredricks, A. Reschly \& S. Christenson (edits.). Handbook of student engagement interventions (pp. 13-29). Londres: Academic Press.

Bailey-Ross, C., Gray, S., Ashby, J., Terras, M., Hudson-Smith, A. \& Warwick, C. (2017). Engaging the museum space: Mobilizing visitor engagement with digital content creation. Digital Scholarship in the Humanities, vol. 32, núm. 4, pp. 689-708. Recuperado de http://dro.dur.ac.uk/20408/

Ballarini, F. (2015). REC. Por qué recordamos lo que recordamos y olvidamos lo que olvidamos. Buenos Aires: Sudamericana.

Bayat, F. (2015). Student engagement: A qualitative study of extracurricular activities. Tesis para obtener el grado de doctor of Education Graduate Department of Leadership, Higher and Adult Education. Ontario Institute for Studies in Education, University of Toronto. Recuperado de https://tspace. library.utoronto.ca/bitstream/1807/70846/1/Bayat_Fraydoon_201511_ EdD_thesis.pdf

Bickmore T., Pfeifer L. \& Schulman D. (2011). Relational agents improve engagement and learning in science museum visitors. En H. H. Vilhjálmsson, S. Kopp, S. Marsella \& K. R. Thórisson (eds.). Intelligent virtual agents (pp. 55-67). Berlin, Heidelberg: Springer. https://doi.org/10.1007/978-3-642-23974-8_7

Blumenfeld, P., Kempler, T. \& Krajcik, J. (2006). Motivation and cognitive engagement in learning environments. En R. K. Sawyer (ed.). The Cambridge handbook of the learning science (pp. 475-488). Nueva York: Cambridge University Press.

Cabral, R. y Maldonado, J. (2019). Museos de ciencias en México: educación y concientización cultural science museums in Mexico: Education and cultural awareness. Naturaleza y Tecnología, vol. 6, núm. 1. Recuperado de www.naturalezaytecnologia.com/index.php/nyt/article/view/343

Castro, D. (2003). Protagonistas de museo: niños, jóvenes y adultos en el desarrollo de proyectos pedagógicos y museográficos sobre el patrimonio arqueológico colombiano. Boletín del Museo del Oro, vol. 51, pp. 2-30. Recuperado de https://publicaciones.banrepcultural.org/index.php/bmo/article/ view/4939

Chávez, K. (2015). Percepciones de los niños visitantes en torno al curso de verano 2014 del Museo Nacional de Culturas Populares, México. Tesis para obtener el grado de licenciado en Turismo. Universidad Autónoma del Estado de México. Recuperado de http://ri.uaemex.mx/handle/20.500.11799/58766

Contreras, J. y Pérez de Lara, N. (2010). Investigar la experiencia educativa. Madrid: Morata.

Corbalán, J. (2008). ¿De qué se habla cuando hablamos de creatividad? Cuadernos de la Facultad de Humanidades y Ciencias Sociales, vol. 35, pp. 11-21.

Corno, L. \& Anderman, E. (2016). Handbook of Educational Psychology. Nueva York: Routledge.

Dindler, C. \& Iversen, O. (2009). Motivation in the museum -mediating between 
everyday engagement and cultural heritage. Conference Paper. Recuperado de http://pure.au.dk/portal/files/108111036/motivation.pdf

Domínguez Arranz, A. (2003). La museología participativa. La función de los educadores de museo. En J. M. Iglesias Gil (edit.). Actas de los XIII Cursos Monográficos sobre Patrimonio Histórico (pp. 99-117). Reinosa. Servicio de $\mathrm{Pu}-$ blicaciones de la Universidad de Cantabria.

Eastwell, P. \& Rennie, L. (2002). Using enrichment and extracurricular activities to influence secondary students' interest and participation in science. The Science Education Review, vol. 1, núm. 4. Recuperado de http://citeseerx.ist. psu.edu/viewdoc/download?doi=10.1.1.524.3433\&rep=rep1\&type=pdf

Eccles, J. S. \& Midgley, C. (1989). Stage_environment fit: Developmentally appropriate classrooms for young adolescents. En C. Ames \& R. Ames (eds.). Research on motivation in education (vol. 3: Goals and cognitions, pp. 13-44). Nueva York: Academic Press.

Elisondo, R. (2015). La creatividad como perspectiva educativa. Cinco ideas para pensar los contextos creativos de enseñanza y aprendizaje. Revista Electrónica Actualidades Investigativas en Educación, vol. 15, núm. 3, pp. 1-23. Recuperado de https://revistas.ucr.ac.cr/index.php/aie/article/view/20904

Falk, J. \& Dierking, L. (2000). Learning from museums. Visitor experiences and the making of meaning. Walnut Creek, Estados Unidos: Altamira Press.

Fletcher, A., Nickerson, P. \& Wright, K. (2003). Structured leisure activities in middle childhood: Links to well-being. Journal Community Phychology, vol. 31, núm. 6, pp. 641-659. https://doi.org/10.1002/jcop.10075

Fredricks, J., Reschly, A. \& Christenson, S. (2019). Interventions for student engagement: Overview and state of the field. En J. Fredricks, A. Reschly \& S. Christenson (edits.). Handbook of Student Engagement Interventions (pp. 1-8). Londres: Academic Press.

Fredricks, J. y Eccles, J. (2008). Participation in Extracurricular Activities in the Middle School Years: Are There Developmental Benefits for African American and European American Youth? Journal of Youth and Adolescence, vol. 37, pp. 1029-1043. Recuperado de https://www.psc.isr.umich.edu/pubs/abs/5750

Freire, P. y Faundez, A. (2013). Por una pedagogía de la pregunta. Critica a una educación basada en respuestas a preguntas inexistentes. Buenos Aires: Siglo Veintiuno.

Leinhardt, G. \& Crowley, K. (1998). Museum learning as conversational elaboration: A proposal to capture, code, and analyze talk in museums. Report \# MLC01. Recuperado de http://mlc.lrdc.pitt.edu/mlc

Melgar, M. F. (2019). ¡Todo me gustó! Los niños y sus percepciones sobre una actividad educativa en un museo. Revista Educatio Siglo XXI, vol. 27, núm. 1, pp. 167-194.

Melgar, M. F y De los Reyes Leoz, J. (2018). De los objetos al museo. Experiencias educativas emotivas y creativas. Monográfico. Revista Contextos de Educación, vol. 25, pp. 122-137.

Melgar, M. F., Elisondo, R. y Donolo, D. (2018). Experiencias en museos. Zonas educativas posibles. Revista Edetania. Estudios y Propuestas Socioeducativas, vol. 53, pp. 241-256. Recuperado de http://revistas.ucv.es/index.php/Edetania/article/view/333/360

Morentin Pascual, M. y Guisasola Aranzabal, J. (2014). La visita a un museo de ciencias en la formación inicial del profesorado de educación primaria. 
Revista Eureka sobre Enseñanza y Divulgación de las Ciencias, vol. 11, núm. 3, pp. 364-380. Recuperado de https://revistas.uca.es/index.php/eureka/ article/view/2890

NCCPE (2015). What is public engagement? Recuperado de https://www.publicengagement.ac.uk/about-engagement/what-public-engagement

O’Brien, E. \& Rollefson, M. (1995). Extracurricular participation and student engagement. Education Policy Issues: Statistical Perspectives. Washington, DC: ERIC Clearinghouse.

Reeve, J., Cheon, S. \& Jang, H. R. (2019). A teacher-focused intervention to enhance students' classroom engagement. En J. Fredricks, A. Reschly \& S. Christenson (eds.). Handbook of student engagement interventions (pp. 87-100). Londres: Academic Press.

Rigo, D. (2017). Docentes, tareas y alumnos en la definición del compromiso. Investigando el aula de nivel primario de educación. Educação em Revista, vol. 33, pp. 1-24. http://dx.doi.org/10.1590/0102-4698154275

Rigo, D., De la Barrera, M. L. y Elisondo, R. (2018a) Investigación educativa... algunos temas de la agenda actual. Revista Contextos de Educación, vol. 25, pp. 159-173. Recuperado de http://www2.hum.unrc.edu.ar/ojs/index.php/ contextos/issue/view/62/showToc

Rigo, D., De la Barrera, M. L. y Elisondo, R. (2018b). La mesa está servida. 4c: cerebro, creatividad, compromiso y contexto. Revista Contextos de Educación, vol. 25, pp. 6-10. Recuperado de http://www2.hum.unrc.edu.ar/ojs/index. $\mathrm{php} /$ contextos/issue/view/62/showToc

Rigo, D. y Donolo, D. (2019). Análisis de un modelo integrador del compromiso escolar: relaciones entre variables situacionales, escolares, sociales y personales en alumnos de nivel primario de educación. Propósitos y Representaciones, vol. 7, pp. e316-316. https://dx.doi.org/10.20511/pyr2019.v7nSPE.316

Rigo, D. y Donolo, D. (2018). ¿Es posible invertir la forma en que aprendemos y enseñamos? Aderezos para repensar la educación. Revista Innovaciones Educativas, vol. XX, núm. 28, pp. 106-119. Recuperado de http://investiga.uned. ac.cr/revistas/index.php/innovaciones/article/view/2135/2501

Shernoff, D. (2013). Optimal learning environments to promote student engagement. Nueva York: Springer.

Templeton, C. \& Baskinger, M. (2011). Museum visitor engagement through resonant, rich and interactive experiences. Tesis para obtener el grado de maestro en Design in Interaction Design. School of Design, Carnegie Mellon University. Recuperado de https://kilthub.cmu.edu/articles/Museum_Visitor_Engagement_Through_Resonant_Rich_and_Interactive_Experiences/6723569/1

Unal, F. (2012). Observation of object preferences of interest by children aged between 4 and 8 in museums: Antalya museum examples. Procedia, vol. 51, pp. 362-367. https://doi.org/10.1016/j.sbspro.2012.08.173

Unesco (2016). Educación 2030. Declaración de Incheon. Hacia una educación inclusiva y equitativa de calidad y un aprendizaje a lo largo de la vida para todos. Recuperado de http://www.unesco.org/new/fileadmin/MULTIMEDIA/ FIELD/Santiago/pdf/ESP-Marco-de-Accion-E2030-aprobado.pdf 